\title{
Differential efatutazone's impact on mammary neoplasia dependent upon Brca1 dose
}

\section{Dear Editor,}

The impact of oral peroxisome proliferator-activated receptor gamma agonist (PPARG) efatutazone exposure through diet on mammary preneoplasia and cancer development was evaluated in genetically engineered mice carrying one vs two disrupted Brca1 alleles targeted to mammary epithelial cells in the background of germline $\operatorname{Trp53}$ (p53) haploinsufficiency. Brca1 ${ }^{\text {WT/fl11//Cre/p53+/- }}$ and Brca1fl11/f11//Cre/p53+/- female mice were randomly assigned to receive efatutazone-containing diet $(30 \mathrm{mg} / \mathrm{kg})$ or equivalent diet without efatutazone at age 2 months. Efatutazone exposure significantly increased the percentage of mice with tertiary branching (Fig. 1A). Palpable mammary cancers appeared significantly earlier in Brca1 fl11/f11//Cre/p53+/- as compared to Brca1 ${ }^{W T / f 11 / / C r e / p 53+/-}$ mice, independent of efatutazone exposure (Fig. 1B). Efatutazone significantly reduced mammosphere development in Brca1 fl11/f11//Cre/p53+/- mice (Fig. 1C, D and E). The significant reduction in numbers and prevalence of hyperplastic alveolar nodules (HANs) was more profound in Brca1 ${ }^{W T / f 11 / C r e / p 53+/-}$ as compared to Brca 1 fl11/fl11//Cre/p53+/mice (Fig. 1F and G). Palpable subcutaneous lipomas appeared in both efatutazone cohorts beginning at age 7 months, significantly shifting the reason for killing in the two different models (Fig. 2A). Fewer Brca1 1 WT/f111/Cre/p53+/mice were killed for palpable mammary gland cancer than Brca1 fl11/f11//Cre/p53+/- mice. Most mice developed one mammary cancer with a range of histology. Four mice developed two palpable mammary cancers: three Brca1 1111/f11//Cre/p53+/- mice (two control and one efatutazone cohort) and one Brca1 ${ }^{\text {WT/f11 } / / \mathrm{Cr} / \mathrm{p} 53+/-}$ mouse (control cohort). Most mammary cancers were triple negative (Fig. 2B, C, D, E, F and G). The two estrogen receptor alpha (ER)- and progesterone receptor (PR)-positive mammary adenocarcinomas that appeared were unique to the

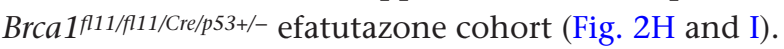

The study was prompted by a previous investigation demonstrating that efatutazone exposure beginning at age 4 months in Brca1 f11/ffl1//Cre/p53+/- mice, a model which typically generates a range of histology of triple-negative mammary cancers, significantly reduces mammary hyperplasia and induces more differentiated mammary cancers including some ER-positive adenocarcinomas (Nakles et al. 2013). Questions raised by the earlier study included whether or not mice carrying a disruption in only one Brca1 allele (Alothman et al. 2017) might be more responsive to efatutazone and the possibility that earlier administration would be more effective in reducing mammary cancer development (Dong 2013). PPARG has been reported as detectable in over $90 \%$ of triple-negative breast cancers developing in women who carry a BRCA1 mutation (Heublein et al. 2017).

Significant differences in efatutazone response were found between the two models. Both models are derived from the same breeding colony with one vs two disrupted Brca1 alleles discriminating between them. The proportion of control mice that developed mammary cancer by age 12 months was not significantly different between the two genotypes; however, the models did exhibit statistically significantly different time-courses of mammary carcinogenesis. Efatutazone was delivered at the same age in both models, raising the possibility that the significant differences in response found between the two models was due to the timing of the intervention relative to the course of mammary carcinogenesis, rather than the underlying genetic difference in full-length Brca1 expression. Even the significant difference in mammosphere development between the models could be related to timing as the published literature suggests that mammosphere development from cancer cells is more profoundly affected by a PPARg agonist as compared to non-cancer cells. Results could argue that for efatutazone to be maximally effective, it may need to be initiated at very early stages of carcinogenesis. Theoretically to test that hypothesis efatutazone could be started even 
A

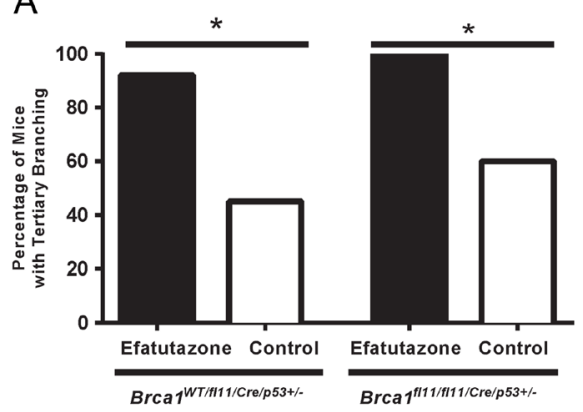

C

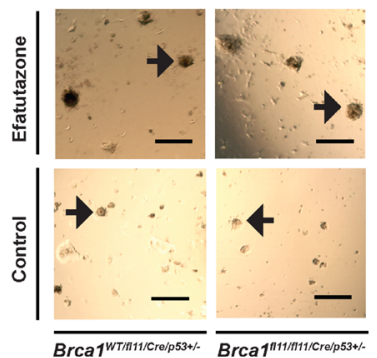

D

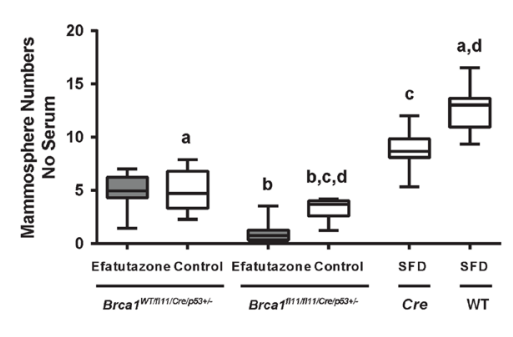

B

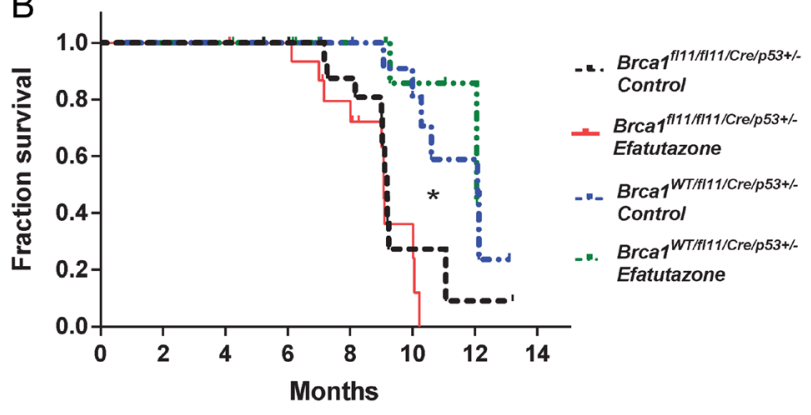

E

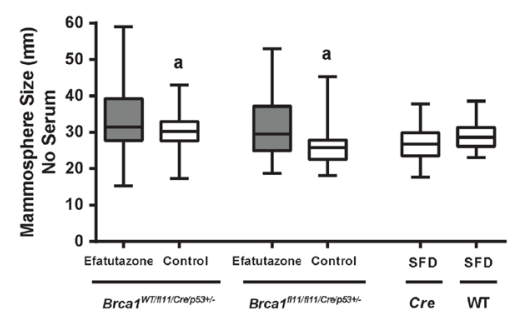

F

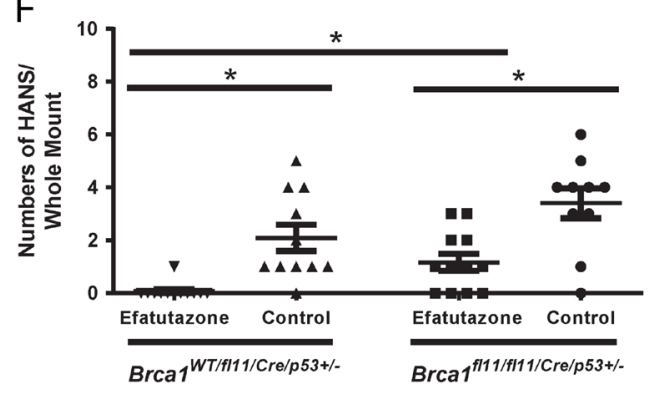

G

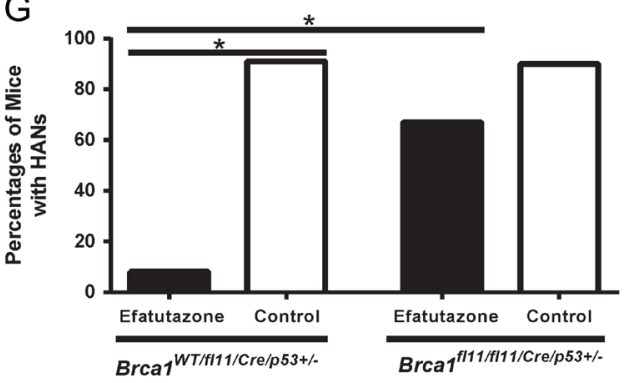

Figure 1

Efatutazone exposure starting at age 2 months significantly reduced mammary preneoplasia. (A) Bar graphs comparing percentages of Brca 1 WT/fl11/Cre/p53+/and Brca $1^{f / 11 / f 111 / C r e / p 53+/-}$ mice demonstrating tertiary branching on fourth (inguinal) mammary gland whole mounts (WMs) following exposure to efatutazone or control diet. ${ }^{*} P<0.05$, Fisher's exact test, one-sided. WM number $(n)$ analyzed, age (months ( $m$ ), mean \pm S.E.M.) and weight (grams (g), mean \pm S.E.M.) for each cohort: Brca 1WT/fl11//re/p53+/- Efatutazone (E): $n=12(9.0 \pm 0.7 \mathrm{~m}, 38.0 \pm 1.9 \mathrm{~g})$. Control $(\mathrm{C}): n=12(10.1 \pm 0.5 \mathrm{~m}, 36.9 \pm 2.4 \mathrm{~g})$. Brca 1 fl11/fl11/cre/p53+/- E: $n=12(8.4 \pm 0.3 \mathrm{~m}$, $40.3 \pm 2.2 \mathrm{~g})$. C: $n=11(8.9 \pm 0.3 \mathrm{~m}, 40.6 \pm 2.4 \mathrm{~g})$. WMs prepared at the time of necropsy for experimental endpoints designated in IACUC-approved protocol. All available fourth gland WMs analyzed. Mean ages and weights at necropsy were not statistically significantly different between cohorts. (B) Kaplan-Meier survival plot of all Brca 1WT/f111/Cre/p53+/- mice on efatutazone (green) and control (blue) and Brca 1f111/f111/Cre/p53+/- mice on efatutazone (red) and control (black) diets enrolled in study. *Log-rank test for trend chi-Square $P=0.0005$ between both Brca 1WT/f111//Cre/p53+/- and Brca $1^{f / 11 / f 111 / C r e / p 53+/-}$ cohorts. Cohort numbers ( $n$ ), median age (m) from survival plot: Brca1wT/f11//Cre/p53+/- E: $n=17$ (12.1 m). C: $n=15$ (12.1 m). Brca1f/11/f111/Cre/p53+/- E: $n=17$ (9.1 m). C: $n=19$ (9.2). Censored points for death due to non-palpable-tumor-related health reasons prior to age 12 months indicated by vertical ticks (skin ulcer, rectal prolapse, leg paralysis, shaking, lymphoma, found dead). Median survival was not statistically significant between efatutazone and control cohorts for either genotype. (C) Representative phase-contrast images of mammospheres (indicated by black arrows) that formed from primary mammary epithelial cells isolated from Brca 1WT/f/11/Cre/p53+/- and Brca 1 f/11/f/11//Cre/p53+/- on E and C diets. Images taken at $4 \times$. Scale bar $=0.1 \mathrm{~mm}$. (D) Box and whisker plots (mean and range) illustrating a reduction in mammosphere numbers forming from primary mammary epithelial cells isolated from efatutazone-exposed Brca $1^{f 111 / f 111 / C r e / p 53+/-}$ mice as compared to Brca $1^{f 111 / f 111 / C r e / p 53+l-}$ mice on control diet. ${ }^{b} P<0.05$ Mann-Whitney $U$ test, two-tailed. Twelve wells plated and analyzed per mouse. Cohort

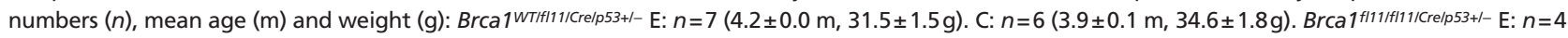

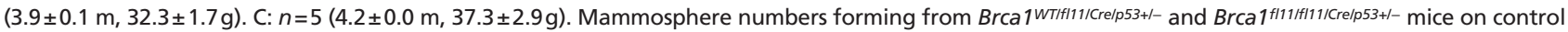
diet were significantly lower than numbers forming from MMTV-Cre (Cre) $\mathrm{c}$ and wild-type (WT) ${ }^{\mathrm{a}, \mathrm{d}}$ mice on standard facility diet. $P<0.05 \mathrm{Kruskal-Wallis} \mathrm{Test,}$ Dunn's multiple comparison. MMTV-Cre: $n=3(4.2 \pm 0.0 \mathrm{~m}, 31.2 \pm 5.1 \mathrm{~g})$. WT: $n=3(5.2 \pm 0.0 \mathrm{~m}, 25.6 \pm 0.6 \mathrm{~g})$. (E) Box and whisker plots (mean and range) illustrating that mammospheres derived from efatutazone-exposed Brca 1 f/11/f/11//Cre/p53+/- were significantly smaller than those obtained from efatutazoneexposed Brca 1WT/f11//Cre/p53+/- mice. a $P<0.05$ Kruskal--Wallis Test, Dunn's multiple comparison. Wells and cohorts analyzed were the same as those analyzed for mammosphere numbers (Panel D). (F) Scatter plots (mean \pm S.E.M. indicated) comparing numbers of HANs per WM from Brca 1 WT/f111//Cre/p53+/- and Brca 1 ${ }^{f 111 / f 11 / / C r e / p 53+/-}$ mice following exposure to efatutazone or control diet. * $P<0.05$, Mann-Whitney $U$ test, one-tailed. WMs and cohorts analyzed were the same as those analyzed for tertiary branching (Panel A). (G) Bar graphs comparing percentages of Brca 1WT/f/11/Cre/p53+/- and Brca 1fl11/f111/Cre/p53+/- mice demonstrating HANs on WM following exposure to efatutazone or control diet. ${ }^{*} P<0.05$, Fisher's exact test, one-sided. WMs and cohorts analyzed were the same as those analyzed for tertiary branching (Panel A). 


\section{A}

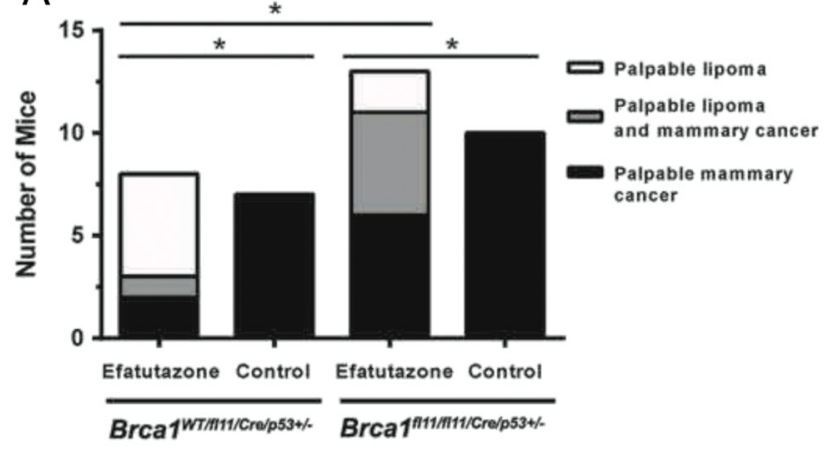

B

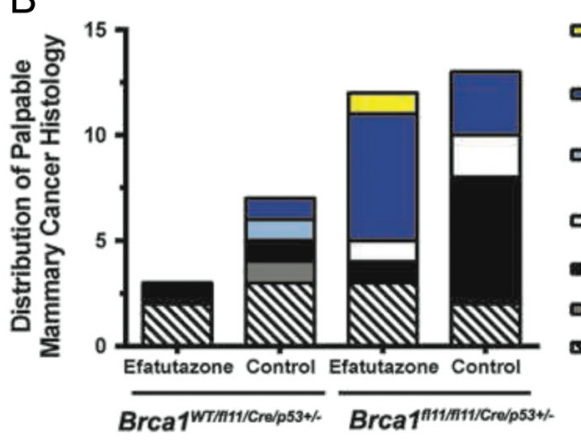

Moderately Differentiated Poorly Differentiated Adenocarcinoma Poorly Differentiated Adenocarcinoma Anth focal Squamous Anaplastic/ Poorly Differentiated Anaplastic Carcinoma Sarcomatoid/ Anaplastic Carcinoma Sarcomatoid Carcinoma
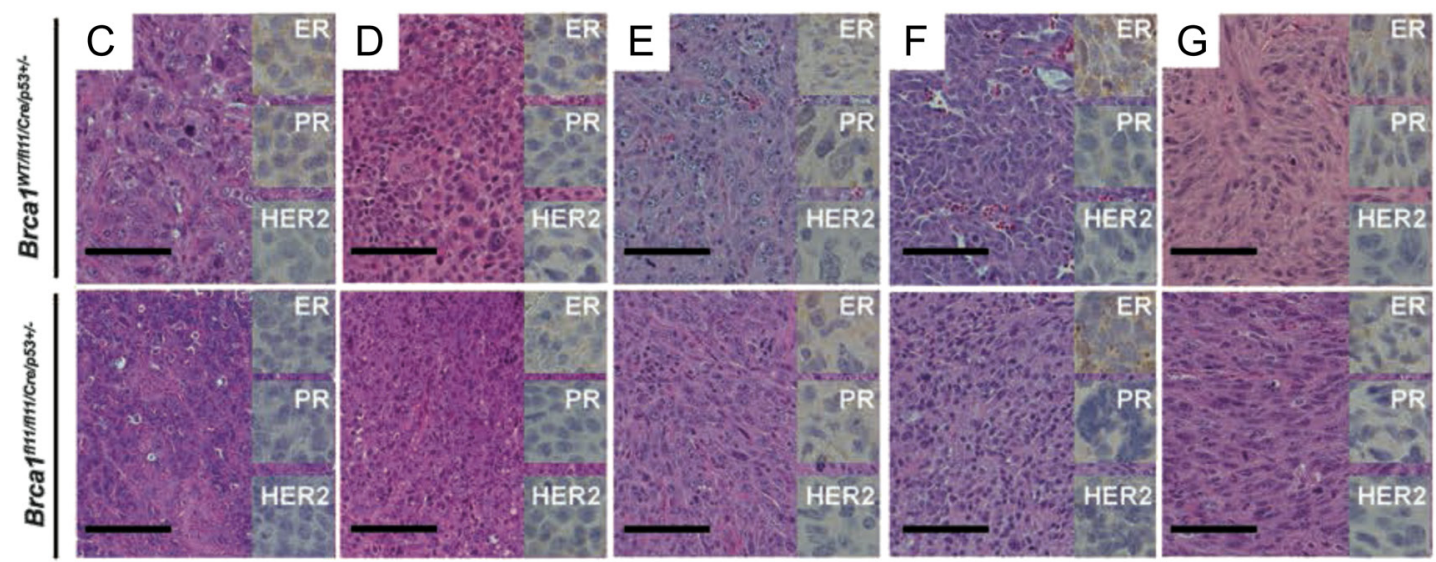

Adenocarcinoma

Anaplastic Carcinoma

Sarcomatoid Carcinoma

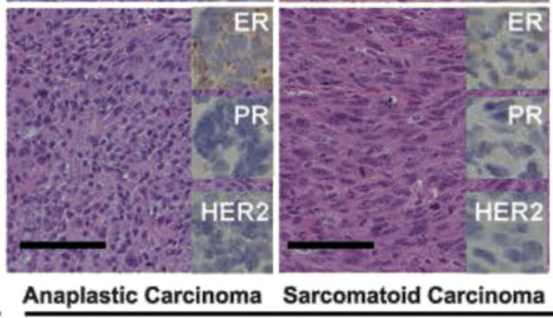

Control

Efatutazone
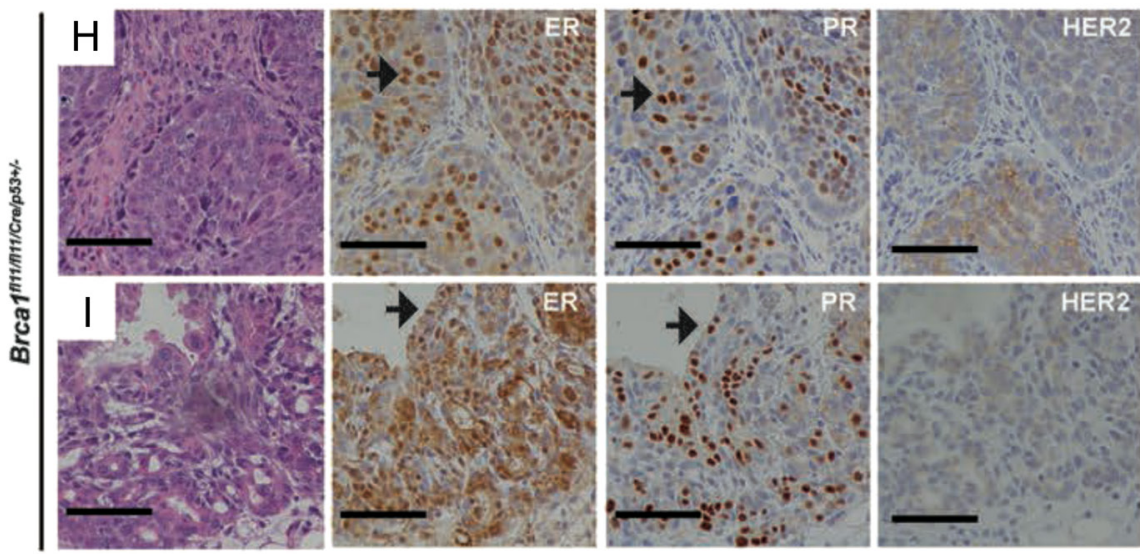

Adenocarcinoma

Efatutazone

\section{Figure 2}

Impact of efatutazone exposure on mammary cancer development in Brca 1WT/f111/Cre/p53+/- and Brca 1fl11/f/11/Cre/p53+/- mice. (A) Stacked bar graphs comparing numbers of mice euthanized for tumor burden with lipoma alone, lipoma and mammary cancer, or mammary cancer alone. ${ }^{*} P<0.05,2 \times 3$ Fisher's exact. Number $(n)$ of mice and age (m, mean \pm s. E.M.) for each cohort: Brca1WT/fl11//Cre/p53+l- E: $n=8$ (9.8 $\pm 0.7 \mathrm{~m})$. C: $n=7$ (10.4 $\pm 0.7 \mathrm{~m})$. Brca 1 fl11/f/11/Cre/p53+/- E: $n=13$ $(8.5 \pm 0.4 \mathrm{~m})$. C: $n=10$. $(9.0 \pm 0.4)$. (B) Stacked bar graphs comparing distribution of mammary cancer histology identified in the palpable mammary

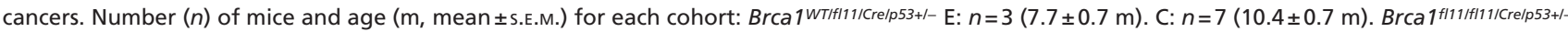
$\mathrm{E}: n=11(8.6 \pm 0.4 \mathrm{~m}) . \mathrm{C}: n=10$. $(9.0 \pm 0.4)$. Representative hematoxylin and eosin images of triple negative mammary adenocarcinoma (C), anaplastic carcinoma (D and F), sarcomatoid carcinoma (E and G) histology from palpable mammary cancers that developed in Brca 1WT/f111/Cre/p53+/- and Brca $1^{f 111 / f 11 / / C r e / p 53+/-}$ mice on efatutazone or control diet. Insets to right show representative images of ER (top), PR (middle) and HER2 (bottom) IHC. ( $H$ and I) Representative images of the histology and ER, PR and HER2 IHC of the two palpable ER+/PR+ mammary adenocarcinomas that developed in

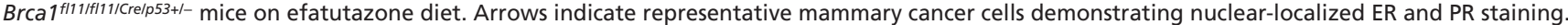

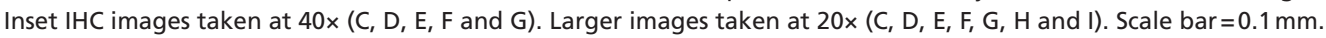


before the end of puberty; however, here we identified a toxicity, lipoma development, prominent in this study but not found when efatutazone exposure was started at age 4 months (Nakles et al. 2013). PPARg activation previously has been suggested as a component of lipoma pathophysiology (Koppen \& Kalkhoven 2010). The study indicates that developmental age at exposure may also play a role. Finally, we cannot exclude the possibility that germ-line Trp53 haploinsufficiency also contributed to the susceptibility of the mice for lipoma development.

For preneoplasia reduction, Brca1 ${ }^{\text {WT/ffl1//Cre/p53+/- mice }}$ were more sensitive than Brcalfl11/f11//Cre//53+/- mice, but this did not translate to absolute mammary cancer prevention. The possibility of a parallel significant reduction in mammary cancer appearance could not be fully assessed due to the unexpected appearance of the palpable lipomas, found uniquely in this study when efatutazone was initiated at the younger age. This necessitated killing for tumor burden, required by the Institutional Animal Use and Care approved protocol, prior to the scheduled endpoint for evaluation of mammary cancer prevalence (age 12 months) in a significant proportion of the

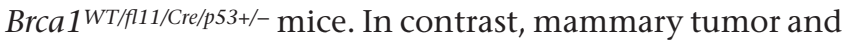
lipoma development appeared temporally coincident in the Brca1f11/ffl1/Cre/p53+/- mice that exhibit earlier mammary cancer development. The appearance of lipomas in Brca1 fl11/f11//Cre/p53+/- mice did not compromise assessment of mammary cancer prevalence, which was unchanged by efatutazone exposure.

An alternative or contributing hypothesis for the significant differences found would be a differential action of efatutazone based on the presence or absence

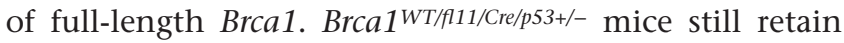
one functional Brca1 allele, and mammary cancer development occurs without loss of the second Brca1 allele (Alothman et al. 2017), as occurs in a portion of women carrying BRCA1 mutation (Roy et al. 2011). PPARG agonists are reported to increase BRCA1 expression in vitro in human breast cancer cells and in vivo in mammary glands of FVB mice (Subbaramaiah et al. 2012). Perhaps significantly, efatutazone is reported to be effective in delaying progression and inducing differentiation in a xenograft model of triple-negative ductal carcinoma in situ (MCFDCIS) with intact BRCA1 and p53 alleles (Ory et al. 2018).

Replicated in this investigation was the novel appearance of $\mathrm{ER}+/ \mathrm{PR}+$ mammary adenocarcinomas in

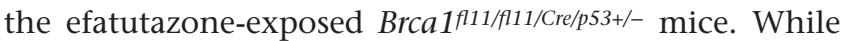
a low-incidence event in both this and the prior study (Nakles et al. 2013), it did appear in two independent and temporally distinct experiments. Results support the possibility that activation of the PPARG pathway can contribute to the appearance of more differentiated mammary cancers retaining expression of ER and PR. None of the cancers expressed pathophysiological levels of Erb-B2 receptor tyrosine kinase 2 (ErbB2/Her2) and the majority of cancers in all groups were triple negative, consistent with previous studies in these and related models (Nakles et al. 2013).

Recognizing the fact that efatutazone was not protective of mammary cancer development, even as it significantly reduced preneoplastic growth, prompts the question of whether or not combining the drug with a second agent such as an antihormonal, a retinoid $\mathrm{X}$ receptor RXR ligand, TNF superfamily member 11 (TNFSF11)/RANKL inhibition or agents affecting the cell cycle such as cyclin-dependent kinase (CDK) $4 / 6$ inhibitors would be more effective. Time-course studies in which interventions are initiated at different ages in models with different temporal patterns of carcinogenesis on a prospective basis may help to further define the points during carcinogenesis when specific preventive agents can be effective. Direct comparison of the mechanistic action of efatutazone in in vitro and in vivo models with different gene dosages of Brca1 and Trp53 intact could establish if there are specific roles for their respective proteins in moderating response to a PPARG agonist.

All animal procedures were performed in accordance with federal guidelines and were approved by the Georgetown University Institutional Animal Care and Use Committee. Generation of mouse cohorts, dietary interventions (F3028, rodent diet, grain-based 1/2-in pellets without or with efatutazone $(30 \mathrm{mg} / \mathrm{kg})$, Bio-Serv, Frenchtown, NJ, USA) and histology were performed as previously described (Nakles et al. 2013, Alothman et al. 2017). Pathology was read blindly by a board-certified pathologist (B V K). NanoCulture Plates (ORGANOGENIX Inc., Kanagawa, JP) were used to assess 3D mammosphere formation in serum-free EpiCult-B Mouse media (STEMCELL Technologies, Vancouver, CA, USA) (Arai et al. 2016, Alothman 2018). Immunohistochemical (IHC) procedures: ER (primary antibodies: ab75635, 1:50, overnight $4^{\circ} \mathrm{C}$, Abcam; 06-935, 1:100, $1 \mathrm{~h}$, room temperature (RT), Millipore Sigma), PR (sc538, 1:350, 1h, RT, Santa Cruz Biotechnology, Inc.), HER2 (ERBB2) (2165, 1:100, overnight $4^{\circ} \mathrm{C}$, Cell Signaling). Before primary antibody exposure, heat-induced epitope retrieval was performed by immersing tissue sections at $98^{\circ} \mathrm{C}$ for $20 \mathrm{~min}$ in $10 \mathrm{mM}$ citrate buffer (pH 6.0) with $0.05 \%$ Tween (ER, PR) or $98^{\circ} \mathrm{C}$ for $20 \mathrm{~min}$ in $0.5 \mathrm{M}$ EDTA 
buffer ( $\mathrm{pH}$ 8.0) (HER2) followed by treatment with $3 \%$ hydrogen peroxide and 10\% normal goat serum (10 min each). Following primary antibody exposure, slides were exposed to an HRP-labeled polymer (EnVision+ SystemHRP Labeled Polymer Anti-Rabbit, K4003, Dako, 30 min) and 3'-Diaminobenzidine (DAB) (Dako, $5 \mathrm{~min}$ ). Cancers were read as $\mathrm{ER}+/ \mathrm{PR}+$ when nuclear localization of both proteins was present. Two independent primary antibodies were used to test ER nuclear localization. Positive (human $\mathrm{ER}+/ \mathrm{PR}+/ \mathrm{HER} 2$-amplified breast cancer) and negative (no primary antibody) controls were performed with each individual IHC experiment. Statistical analyses were performed using GraphPad Prism and the VassarStats: Statistical Computation Web Site. $P<0.05$ was considered significant.

Sahar J Alothman ${ }^{1,2}$
Weisheng Wang
Shan Chao ${ }^{1,2}$
Bhaskar V Kallakury
Edgar S Díaz-Cruz
Priscilla A Furth
2,4,6

${ }^{1}$ Graduate School of Arts and Science, Georgetown University, Washington, District of Columbia, USA ${ }^{2}$ Department of Oncology, Georgetown University, Washington, District of Columbia, USA 3 Department of Pathology, Georgetown University, Washington, District of Columbia, USA ${ }^{4}$ Lombardi Comprehensive Cancer Center, Georgetown University, Washington, District of Columbia, USA ${ }^{5}$ College of Pharmacy, Belmont University, Nashville, Tennessee, USA

${ }^{6}$ Department of Medicine, Georgetown University, Washington, District of Columbia, USA

(Correspondence should be addressed to P A Furth: paf3@georgetown.edu)

\section{Declaration of interest}

The authors declare that there is no conflict of interest that could be perceived as prejudicing the impartiality of this article.

\section{Funding}

This work was supported by National Institutes of Health, National Cancer Institute RO1 CA112176 (P A F) and 5P30CA051008 (Histopathology and Tissue and Animal Model Shared Resources) and the King Abdullah Scholarship Program, Ministry of Education, Kingdom of Saudi Arabia.

\section{Author contribution statement}

Study conception and design (S J A, P A F), mouse husbandry (P A F, W W), data procurement (S J A, W W, S C, B V K, E S D-C, P A F), data analysis (S J A, $S C, P A F$ ), writing manuscript ( $\mathcal{A} A, P A F$ ), review and editing manuscript (S J A, W W, S C, B V K, E S D-C, P A F).

\section{References}

Alothman SJ 2018 Studies of Breast Cancer Chemoprevention in Human High-Risk Primary Cells and Genetically Engineered Mouse Models. Washington, DC, USA: Georgetown University. (available at: http:// hdl.handle.net/10822/1050904)

Alothman SJ, Wang W, Goerlitz DS, Islam M, Zhong X, Kishore A Azhar RI, Kallakury BV \& Furth PA 2017 Responsiveness of Brca1 and Trp53 deficiency-induced mammary preneoplasia to selective estrogen modulators versus an aromatase inhibitor in Mus musculus. Cancer Prevention Research 10 244-254. (https://doi.org/10.1158/19406207.CAPR-16-0268)

Arai K, Eguchi T, Rahman MM, Sakamoto R, Masuda N, Nakatsura T, Calderwood SK, Kozaki K-I \& Itoh M 2016 A novel high-throughput 3D screening system for EMT inhibitors: a pilot screening discovered the EMT inhibitory activity of CDK2 inhibitor SU9516. PLOS ONE 11 e0162394. (https://doi.org/10.1371/journal.pone.0162394)

Dong JT 2013 Anticancer activities of PPAR $\gamma$ in breast cancer are context-dependent. American Journal of Pathology 182 1972-1975. (https://doi.org/10.1016/j.ajpath.2013.03.005)

Heublein S, Mayr D, Meindl A, Kircher A, Jeschke U \& Ditsch N 2017 Vitamin $\mathrm{D}$ receptor, retinoid $\mathrm{X}$ receptor and peroxisome proliferatoractivated receptor $\gamma$ are overexpressed in BRCA1 mutated breast cancer and predict prognosis. Journal of Experimental and Clinical Cancer Research 36 57. (https://doi.org/10.1186/s13046-017-0517-1)

Koppen A \& Kalkhoven E 2010 Brown vs white adipocytes: the PPARgamma coregulator story. FEBS Letters 584 3250-3259. (https:// doi.org/10.1016/j.febslet.2010.06.035)

Nakles RE, Kallakury BV \& Furth PA 2013 The PPARgamma agonist efatutazone increases the spectrum of well-differentiated mammary cancer subtypes initiated by loss of full-length BRCA1 in association with TP53 haploinsufficiency. American Journal of Pathology 182 1976-1985. (https://doi.org/10.1016/j. ajpath.2013.02.006)

Ory V, Keitzman W, Boekelman J, Kallakury B, Wellstein A, Furth PA \& Riegel AT 2018 The PPAR $\gamma$ agonist efatutazone delays invasive progression and induces differentiation of ductal carcinoma in situ (DCIS). Breast Cancer Research and Treatment 169 47-57. (https://doi. org/10.1007/s10549-017-4649-y)

Roy R, Chun J \& Powell SN 2011 BRCA1 and BRCA2: different roles in a common pathway of genome protection. Nature Reviews Cancer 12 68-78. (https://doi.org/10.1038/nrc3181)

Subbaramaiah K, Howe LR, Zhou XK, Yang P, Hudis CA, Levy Kopelovich L \& Dannenberg AJ 2012 Pioglitazone, a PPARg agonist, suppresses CYP19 transcription: evidence for involvement of 15-hydroxyprostaglandin dehydrogenase and BRCA1. Cancer Prevention Research 5 1183-1194. (https://doi.org/10.1158/1940-6207. CAPR-12-0201

Received in final form 29 June 2018

Accepted 12 July 2018 (c) 2018 Society for Endocrinology Published by Bioscientifica Ltd. Printed in Great Britain 